\title{
ARTICLE \\ Continuous medium exchange and optically induced electroporation of cells in an integrated microfluidic system
}

\author{
Gwo-Bin Lee ${ }^{1,2,3}$, Chia-Jung Chang ${ }^{1}$, Chih-Hung Wang ${ }^{1}$, Ming-Yu Lu ${ }^{1}$ and Yen-Yi Luo ${ }^{1}$
}

Optically induced electroporation (OIE) is a promising microfluidic-based approach for the electroporation of cell membranes. However, previously proposed microfluidic cell-electroporation devices required tedious sample pre-treatment steps, specifically, periodic media exchange. To enable the use of this OIE process in a practical protocol, we developed a new design for a microfluidic device that can perform continuous OIE; i.e., it is capable of automatically replacing the culture medium with electroporation buffers. Integrating medium exchanges on-chip with OIE minimises critical issues such as cell loss and damage, both of which are common in traditional, centrifuge-based approaches. Most importantly, our new system is suitable for handling small or rare cell populations. Two medium exchange modules, including a micropost array railing structure and a deterministic lateral displacement structure, were first adopted and optimised for medium exchange and then integrated with the OIE module. The efficacy of these integrated microfluidic systems was demonstrated by transfecting an enhanced green fluorescent protein (EGFP) plasmid into human embryonic kidney 293T cells, with an efficiency of $8.3 \%$. This result is the highest efficiency reported for any existing OIE-based microfluidic system. In addition, successful co-transfections of three distinct plasmids (EGFP, DsRed and ECFP) into cells were successfully achieved. Hence, we demonstrated that this system is capable of automatically performing multiple gene transfections into mammalian cells.

Keywords: electroporation; medium exchange; microfluidic; transfection

Microsystems \& Nanoengineering (2015) 1, 15007; doi:10.1038/micronano.2015.7; Published online: 15 June 2015

\section{INTRODUCTION}

In the past few decades, single-function microfluidic components capable of performing specific tasks, such as cell separation $^{1-4}$, particle/cell focusing ${ }^{5}$ and cell electroporation ${ }^{6-8}$, have been thoroughly investigated as stand-alone components in a variety of microfluidic applications. However, in terms of replacing a traditional clinical laboratory with a single lab-on-a-chip, most of these stand-alone microfluidic components were not sufficient to address practical problems that require multiple processing steps. For example, in electroporation, mammalian cells must be transferred from the original culture medium into a specialised electroporation buffer to optimise the electroporation yield ${ }^{9-11}$. Existing microfluidic electroporation devices are incapable of performing this sample pre-treatment step, and the inherent advantages of microfluidic lab-on-a-chip systems, such as automation, user friendliness and the reduction of human error, were compromised. Therefore, a real need exists to develop a microscale total analysis system integrated with different functional microfluidic components to truly leverage the advantages of the aforementioned microfluidic systems.

The cell membrane acts as a barrier to prevent intracellular molecules from randomly being delivered into the interior of cells. Conventional cell electroporation disrupts the integrity of the cell membrane using a short electrical pulse. Electroporation increases the permeability of the cell membrane to allow chemicals, drugs or foreign DNA chains, to enter into cells for biological and medical applications. For successful cell electroporation, the target adherent cells also must be trypsinised, washed, and then transferred into a specialised electroporation buffer to optimise electroporation yield. Furthermore, because of the relatively large size of the electroporation apparatus, a high voltage is commonly used to generate the required electric field for electroporation. This high voltage and the relatively complicated protocol can easily damage the target cells; this protocol also presents a risk of injury to the operator. Hence, the cell viability rate after traditional electroporation is typically less than $50 \%$ for mammalian cell lines ${ }^{12}$.

Recently, electroporation was integrated into microfluidic devices by using various types of mechanisms ${ }^{13-17}$. In comparison to conventional electroporation methods, microfluidic systems have some advantages, including the use of a lower applied electric field, lower sample and reagent consumption and reduced Joule heating ${ }^{6}$. However, existing microfluidic electroporation devices with fixed micro-electrodes are incapable of exchanging media in a sample pre-treatment step. In addition, the fabrication of delicate microelectrodes may also hinder their practical application.

Optically induced electroporation (OIE), also known as lightinduced electroporation, is a microfluidic approach for cell electroporation that offers significant flexibility with respect to other methods requiring fixed metal electrodes ${ }^{15}$. The OIE system enables the operator to quickly reconfigure the electrode geometries and locations to perform different electroporation applications without having to re-fabricate the entire chip ${ }^{18}$. Multiple single cells can be optically targeted for electroporation in parallel by dynamically adjusting the "virtual electrodes" projected by the digitally controlled light patterns. This precision control of the electrode leaves the other cells unaffected. External substances, such

${ }^{1}$ Department of Power Mechanical Engineering, National Tsing Hua University, Hsinchu 30013; ${ }^{2}$ Institute of Biomedical Engineering, National Tsing Hua University, Hsinchu 30013 and ${ }^{3}$ Institute of NanoEngineering and Microsystems, National Tsing Hua University, Hsinchu 30013

Correspondence: Gwo-Bin Lee (gwobin@pme.nthu.edu.tw)

Received: 8 January 2015; Revised: 30 March 2015; Accepted: 22 April 2015 
as fluorescent dyes and genes, have been successfully transferred into mammalian cells using this method ${ }^{15,19}$. However, a proper electroporation buffer (such as a $0.2 \mathrm{M}$ sucrose solution) is commonly used to generate sufficient transmembrane potentials on cells for $\mathrm{OIE}^{19}$. Therefore, the re-suspended cells must be centrifuged in advance to replace the original culture medium with the proper electroporation buffer. Thus, this centrifuging step makes cell electroporation a complicated process.

Microfluidic-based devices for cell separation can be divided into two categories: active or passive approaches. Active microfluidic devices require an external force to control fluid transfer and for cell separation. For example, we previously reported a microfiltering structure to collect cells for the subsequent optically induced cell separation process ${ }^{20}$. Up to $96.2 \%$ of the live cells loaded into the microfluidic device were successfully recovered. However, this device required a precision micropump for flow control. Alternatively, several passive microfluidic approaches to sort and to manipulate cells have been demonstrated previously. For example, a deterministic lateral displacement (DLD) structure is a promising microfluidic technique to separate particles and cells based on their sizes, shape and deformability ${ }^{21-23}$. DLD is a passive, hydrodynamics-based particle-sorting technique that consists of a symmetric array of micro-posts ${ }^{22,24}$. The hydrodynamic interaction between particles and the microstructures enables the separation of particles with different sizes. A larger particle is deflected at a specific angle and then transferred to another flow stream. By contrast, a smaller particle is not affected by these interactions and hence is not laterally displaced. In addition, DLD is able to steer, refract and focus streams of biomaterials and has been implemented in various biomedical applications ${ }^{23,25-30}$. A micro-post array railing ( $\mu$ PAR) structure is another passive, hydrodynamics-based particle-sorting technique $^{31}$. The $\mu$ PAR structure consists of an array of square micro-posts that are tilted at a specific angle with respect to the fluid flow direction. The gaps between micro-posts are smaller than the suspended target particles or cells and could therefore guide them into discrete, adjacent laminar flow streams. This structure had been demonstrated to automatically perform molecular synthesis processes on polystyrene microbead substrates while simultaneously mixing suspended cells with discrete, parallel flow streams ${ }^{31,32}$. Note that the DLD or $\mu$ PAR microstructures were originally demonstrated for the separation of particles or cells. In this work, DLD or $\mu$ PAR microstructures have been adopted to guide cells into a certain direction so that medium exchange could be performed continuously.

Herein, a novel microfluidic device was developed that combines both OIE and medium exchange functions into an integrated system. The continuous medium exchange and electroporation of the cells is performed by the medium exchange modules consisting of $\mu$ PAR or DLD structures and the OIE module, respectively. This system enables a completely integrated cell electroporation workflow. Finally, single or cotransfection of three types of fluorescently labelled plasmids into HEK 293 T cells was successfully demonstrated using this OIE microfluidic system. These results show that this microfluidic system is a promising tool for genetic engineering.

\section{MATERIALS AND METHODS}

\section{Design of the integrated microfluidic system}

The developed integrated microfluidic system has two modules connected in series: the medium exchange and the OIE modules, as schematically shown in Figure 1. First, the re-suspended cells in the original culture medium were transferred into the electroporation buffer by using one of the passive medium exchange modules. Next, the electroporation buffer carrying the cells flows into the OIE module, where electroporation occurs. a

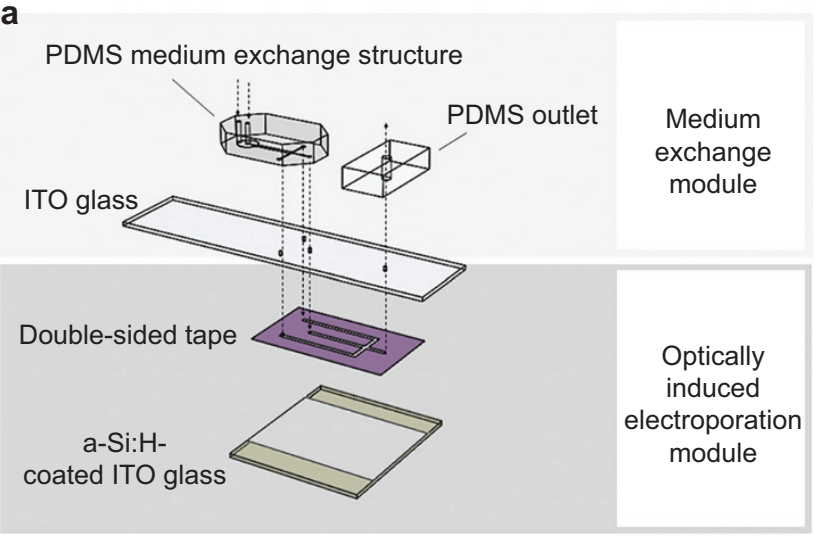

b

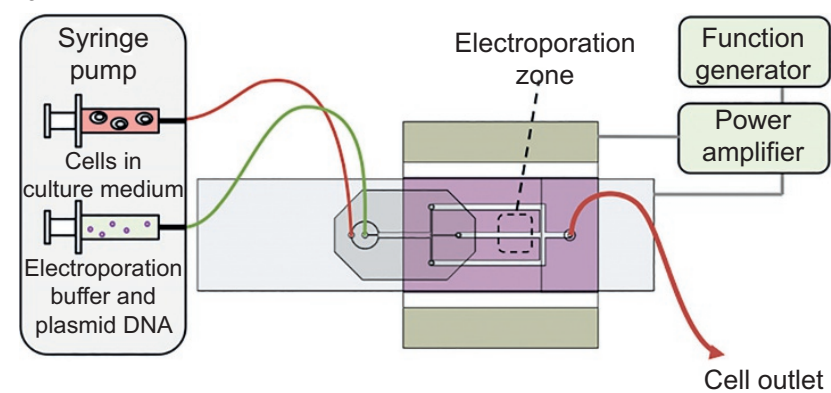

C

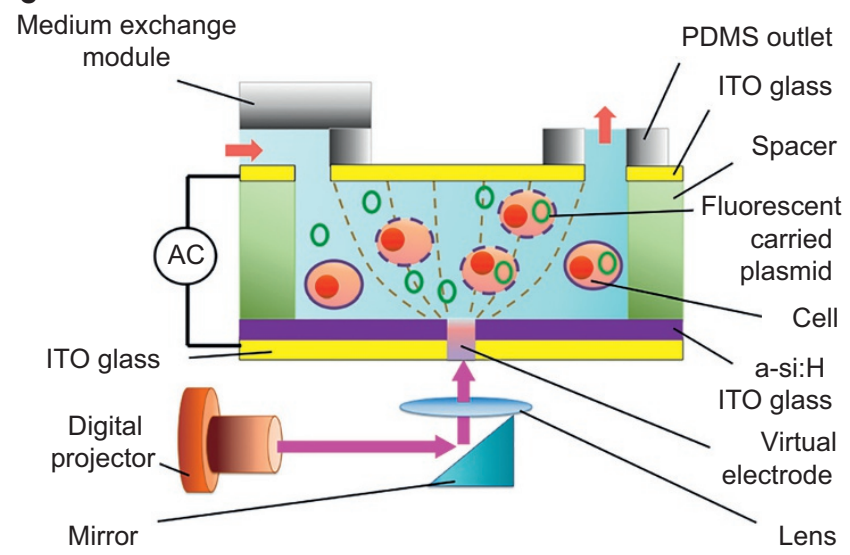

Figure 1 (a) The integrated microfluidic device was composed of two modules: a continuous medium exchange module and an OIE module. The continuous medium exchange module consisted of a patterned PDMS structure bonded to a layer of glass substrate. The OIE module was formed by bonding a top layer of ITO glass to a bottom layer of a-Si:H coated ITO glass with double-sided tape that is patterned by a $\mathrm{CO}_{2}$ laser. The fluid channels in both modules were $50 \mu \mathrm{m}$ in height. The modules were connected by through holes drilled at the interface between the two modules. The processed fluid was collected from the single PDMS outlet located at the rear of the integrated device. (b) Syringe pumps were used to inject the cells suspended in the culture medium and the electroporation buffer to flow through the medium exchange module. Next, the cells were transferred into the electroporation buffer stream (which contained the plasmid DNA), electroporated in the OIE zone in the OIE module and then collected at the outlet. (c) The working principle of OIEbased microfluidic chip. The target cell and fluorescently labelled plasmid were transported into the OIE module. A digital projector illuminated a light spot onto the bottom of a-Si:H layer, which generated a virtual electrode. Next, the fluorescently labelled plasmids were electroporated into the target cell. Finally, the electroporated cells were collected at the outlet and cultured for further analysis. 
As schematically shown in Figure $1 \mathrm{a}$, the continuously flowing, passive medium exchange module consisted of a patterned polydimethylsiloxane (PDMS) structure bonded to a layer of indium-tin-oxide (ITO) glass substrate. The OIE module was formed by bonding the top layer of ITO glass to a bottom layer of hydrogen-rich amorphous-silicon (a-Si:H)-coated ITO glass with double-sided tape patterned by a $\mathrm{CO}_{2}$ laser. The modules were connected via through holes drilled at the interface between the two modules while the processed fluid was collected from the single PDMS outlet located at the top of the integrated device.

\section{Experimental setup}

An illustration of the OIE chip is shown in Figure $1 \mathrm{~b}$. The developed chip was mounted on an upright fluorescence microscope (BX43, Olympus Co., Japan) that allowed for realtime observation using a charge-coupled device (CCD) camera (Evolution VF, Media Cybernetics, Inc., USA). Two fluid streams of (1) medium with cells and (2) electroporation buffer with fluorescently labelled plasmids were continuously injected into the microfluidic chip using two syringe pumps (KDS-270, KD Scientific, USA). Note that double-side tape was used as a spacer. Cells, reagents and buffers were first introduced from the through-hole of the PDMS inlet, and then the entire process, i.e., medium exchange and OIE was performed automatically. The position of the virtual electrodes was determined by the projected light pattern, which was generated by the digital projector. The re-suspended cells were transferred from the culture media into the flowing electroporation buffer using DLD or $\mu$ PAR structures. Figure 1c shows a cross-sectional view of the device that describes the working principle of the optically induced gene transfection. The target cell and fluorescently labelled plasmid were transported via the electroporation buffer into the lower OIE module. A digital projector (PLC-XU106, SANYO Electric Co., Japan) illuminated a light spot onto the a-Si:H layer, which acted as a virtual electrode in the electroporation zone. The alternating-current (AC) voltage was generated by a function generator (AFG-2125, Good Will Instrument Co., Ltd., Taibei) and amplified by a power amplifier (HA-405, PINTEK, New Taipei City). This established an AC electric field between the top and bottom ITO layers. Next, the fluorescently labelled plasmids were electroporated into the target cell. The output voltage was monitored using an oscilloscope (GDS-1102-U, Good Will Instrument Co., Kaohsiung). Finally, the electroporated cells were collected at the outlet and cultured for further analysis.

\section{Design of medium exchange modules: DLD and $\mu$ PAR}

To transfer the cells from the original culture medium into the electroporation buffer, the culture medium with suspended cells was injected into the outer flow. The target cell was guided to the central stream of the electroporation buffer by the DLD structures (Figure 2a). The DLD design parameters, $\lambda=60 \mu \mathrm{m}, \varepsilon=0.05$, gap $=30 \mu \mathrm{m}$, correspond to a critical particle diameter (Dc) of $\sim 9 \mu \mathrm{m}$ and the scanning electron microscope (SEM) image of the DLD microstructure is presented in Figure $2 \mathrm{~b}$. Therefore, any target HEK 293T cells greater than $9 \mu \mathrm{m}$ in diameter fell into the "bump mode" category and could be theoretically transferred into the buffer stream ${ }^{22}$.

For the MPAR structure, the re-suspended cells in the outer streams were guided into the centre of the electroporation buffer stream by the $\mu P A R$ structure (Figure $2 \mathrm{c}$ ). Next, the cells were electroporated using OIE. The $\mathrm{PPAR}$ structure consisted of tilted arrays of rectangular micro-posts with gaps smaller than the diameter of the cells. The length of the sides of the micro-posts was $20 \mu \mathrm{m}$, and the gap between micro-posts was $10 \mu \mathrm{m}$. The tilt angle of the arrays with respect to the fluid flow, $\alpha$, was $1^{\circ}$, and the SEM image of the MPAR microstructure is presented in Figure $2 \mathrm{~d}$.

\section{Fabrication of the medium exchange modules}

The medium exchange modules were micro-fabricated by bonding PDMS fluid channels onto the non-conducting side of the ITO glass substrate. A $50 \mu \mathrm{m}$ thick layer of negative photoresist SU-8 3050 (MicroChem Corp., USA) was photolithographically patterned as a mould and was used to replicate the inverse structure in PDMS. First, a bare silicon wafer substrate was rinsed with double-distilled water $\left(\mathrm{ddH}_{2} \mathrm{O}\right)$ and then sonicated for $5 \mathrm{~min}$ in acetone to detach any particles from the surface. Next, the treated wafer was rinsed with $d_{d d H_{2}} \mathrm{O}$ and then air dried. Afterwards, the wafer was treated with a buffered-oxide-etch (BOE) reagent, rinsed with $\mathrm{dd}_{2} \mathrm{O}$ and then dried again. Following the $\mathrm{BOE}$ treatment, the wafer was placed on a hotplate at $150{ }^{\circ} \mathrm{C}$ for 5 min to dehydrate the surface. After this substrate pre-treatment, the wafer was cooled down to room temperature, and then a layer of SU-8 50 with a thickness of $50 \mu \mathrm{m}$ was spin-coated onto the surface using a spin-coater (200F, Filmtronics, USA). Next, the SU-8 was soft-baked at $65^{\circ} \mathrm{C}$ for $6 \mathrm{~min}$ and then at $95^{\circ} \mathrm{C}$ for $20 \mathrm{~min}$ to evaporate the solvent prior to exposure. Next, the wafer was cooled down to room temperature prior to exposure. The ultraviolet light exposure

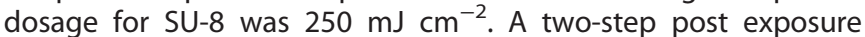
bake was performed immediately after the exposure at $65^{\circ} \mathrm{C}$ for $1 \mathrm{~min}$ and then at $95^{\circ} \mathrm{C}$ for $5 \mathrm{~min}$. Finally, the developed pattern was rinsed with isopropyl alcohol and dried. As a final step, the wafer and SU-8 mould were placed on a hot plate at $175^{\circ} \mathrm{C}$ for 4 hours to enhance the strength of the structure. This structure served as a negative mould for the PDMS. For casting the PDMS, an elastomer (Sylgard 184A silicon elastomer, Dow Corning, USA) and a curing agent (Sylgard 184B elastomer curing agent, Dow Corning, USA) were well mixed in a 10:1 ratio by weight, poured onto the SU-8 mould and then cured at $80^{\circ} \mathrm{C}$ over $2 \mathrm{~h}$ to form the DLD and $\mathrm{PPAR}$ microstructures for medium exchange.

\section{Fabrication of the OIE module and the integration of the two modules}

The OIE channel was patterned on a piece of double-sided tape (50 $\mu \mathrm{m}$ in height; Tesa Tape, Inc., USA) by using a laser-cutting machine (VersaLASER VL-200, Universal Laser Systems, Inc., USA). The patterned tape was attached to the a-Si:H coated ITO glass, and then through-holes were drilled to form an OIE module. Finally, the drilled holes in the PDMS medium exchange structure and the PDMS outlet were aligned and bonded onto the top surface of the ITO glass via oxygen plasma treatment.

\section{Preparation of human embryonic kidney 293T (HEK 293T) cells and electroporation buffer}

Human embryonic kidney cells (HEK 293T, ATCC ${ }^{\circledR}$ CRL-11268TM) were provided by the Institute of Microbiology and Immunology, College of Medicine, Chung Shan Medical University, Taichung and cultured in Dulbecco's Modified Eagle Medium (DMEM), which contained 10\% fetal bovine serum (Sigma Co., USA), 2 mM of L-glutamine (Sigma Co., USA) and 1\% penicillin/streptomycin. The monolayer of cells was trypsinised with $1 x$ trypsin-EDTA (Sigma Co., USA) and then re-suspended in DMEM, yielding a final concentration of 106 cells $\mathrm{ml}^{-1}$. The pEGFP-N1 (4.7 kp), pDsRED (4.1 kp) and pECFP (4.1 kp) plasmids were provided by the Institute of Biomedical Sciences, National Sun Yat-Sen University, Kaohsiung. Note that the three fluorescently labelled plasmid DNAs were extracted with NucleoBond ${ }^{\circledR}$ Xtra Midi kits (MACHEREY 

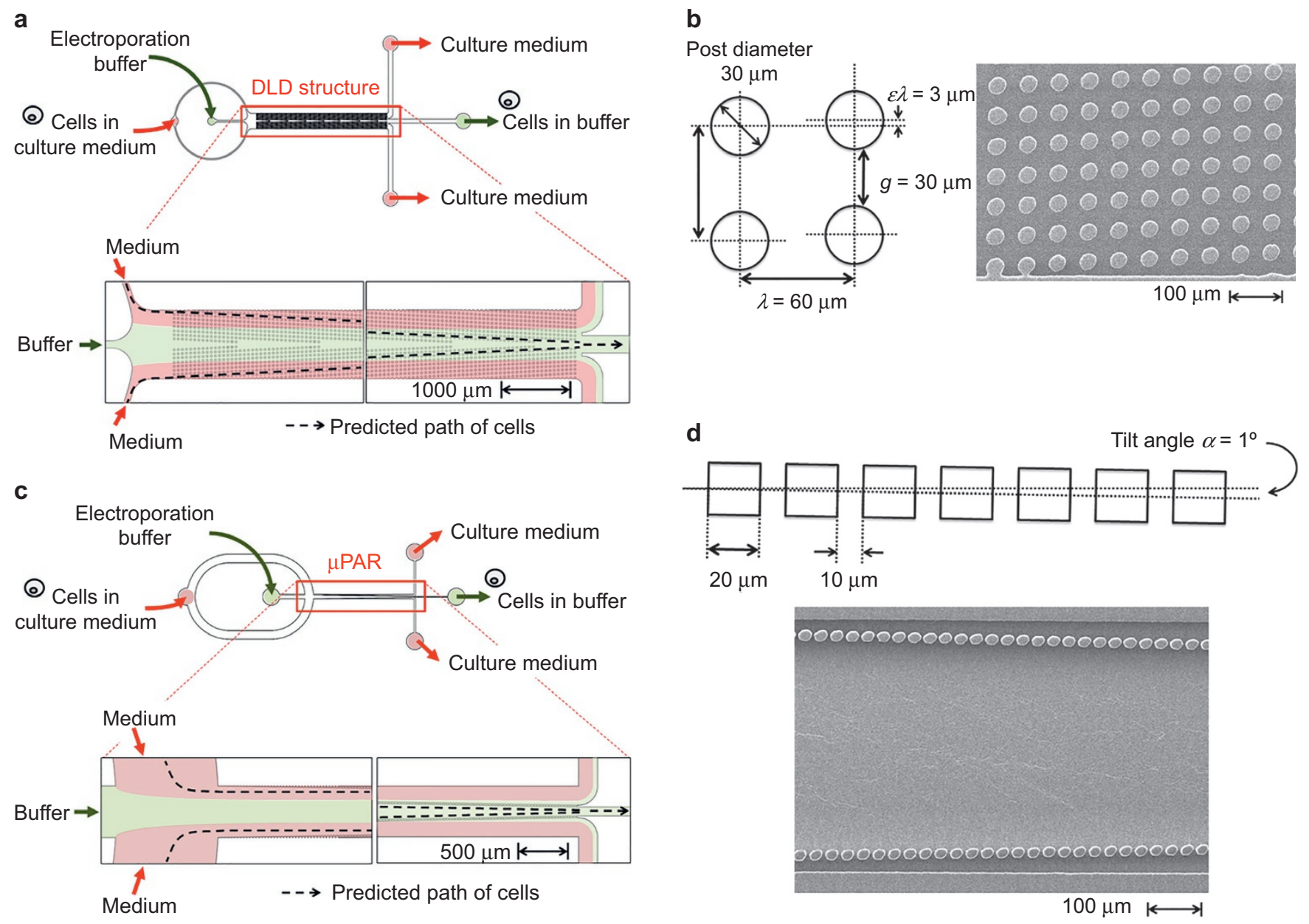

Figure 2 (a) Cells suspended in the culture medium were introduced into the medium exchange module consisting of a DLD structure and then were "bumped" into the central stream of the electroporation buffer. The dashed black lines represent the predicted path of the cells in the DLD structure. (b) The proposed DLD design parameters and a SEM image of the PDMS mould replica. Each post diameter is $30 \mu \mathrm{m}, \lambda$ is $60 \mu \mathrm{m}, \varepsilon$ is 0.05 , and the gap between posts is $30 \mu \mathrm{m}$. The estimated critical particle diameter is approximately $9 \mu \mathrm{m}$. (c) Cells in the culture medium were introduced into the $\mu$ PAR structure and then were guided into the central stream of the electroporation buffer. The dashed black lines are the predicted paths of cells in the $\mu$ PAR structure. (d) Proposed $\mu$ PAR design parameters and an SEM image of the PDMS mould replica. The length of the long side of each rectangular microposts is $20 \mu \mathrm{m}$, the gap between microposts is $10 \mu \mathrm{m}$, and the tilt angle is $1^{\circ}$.

NAGEL GmbH \& Co, Germany), following the protocols in the user's manual. These extracted plasmid DNAs were re-suspended in $0.2 \mathrm{M}$ sucrose (Sigma Co., USA) at a final concentration of $2 \mu \mathrm{g} \mathrm{m}{ }^{-1}$.

\section{OIE parameters}

The optimised experimental parameters for the continuous medium exchange and the OIE experiment are presented in Table 1, which were based on the OIE simulations and the

Table 1 The operating conditions for continuous medium exchange and for the OIE experiments

\begin{tabular}{ll}
\hline Controlled parameters & Value (unit) \\
\hline Applied AC frequency & $20(\mathrm{kHz})$ \\
Light irradiance & $1.5\left(\mathrm{~W} \mathrm{~cm}{ }^{-2}\right)$ \\
Size of "virtual electrode" & $3.5 \mathrm{~mm} \times 3.0 \mathrm{~mm}$ \\
\hline
\end{tabular}

practical operating limits of the instrumentation. Specifically, the applied AC frequency was designated to be just below the value that would cause hydrolysis in the OIE channel, and the light irradiance and light spot size were both set at stable conditions near the upper limits of the optical system.

\section{RESULTS}

\section{Exchange efficiency of the medium exchange modules}

Tests on the medium exchange modules were first performed to ensure the successful performance of the proposed device. The deflection of the cellular trajectory was observed in the DLD continuous medium exchange module (Figure 3a). The HEK 293T cells in the DLD structure were in the "bumping mode", where the trajectory of the cells was inclined at a specific tilt angle towards the centre of the channel, resulting in an overall lateral displacement that shifted the cells from the outer flow to the centre flow of the electroporation buffer. Note that the medium and buffer flows were injected using syringe pumps at the same flow rates of $25 \mu \mathrm{l} \mathrm{min}{ }^{-1}$. For the medium exchange module with 
a

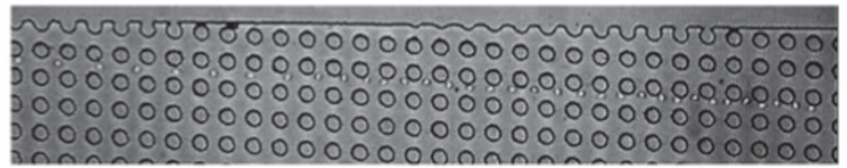

Flow direction

central outlet by the number of total cells collected from all three outlets (as shown in Equation (1)).

$$
\begin{aligned}
\text { Exchange efficiency }= & \frac{\text { Cells collected from the central outlet }}{\text { Total number of collected cells }} \\
& \times 100 \%
\end{aligned}
$$

The experimental results showed that the exchange efficiency was higher for the proposed $\mu$ PAR module than the DLD module at different fluid flow rates. For the DLD medium exchange module, the exchange efficiency increased with increasing flow rate and saturated at high flow rates (Figure $4 a$ ). The highest exchange efficiency of $33.7 \%$ was observed at a volume flow rate of $90 \mu \mathrm{l} \mathrm{min}-1$ (Figure $4 \mathrm{a}$ ). In the $\mu$ PAR medium exchange module, the exchange efficiency also increased with increasing flow rates. The stronger the vacuum suction, the faster the fluid flow and the higher the exchange efficiency. Saturation was also
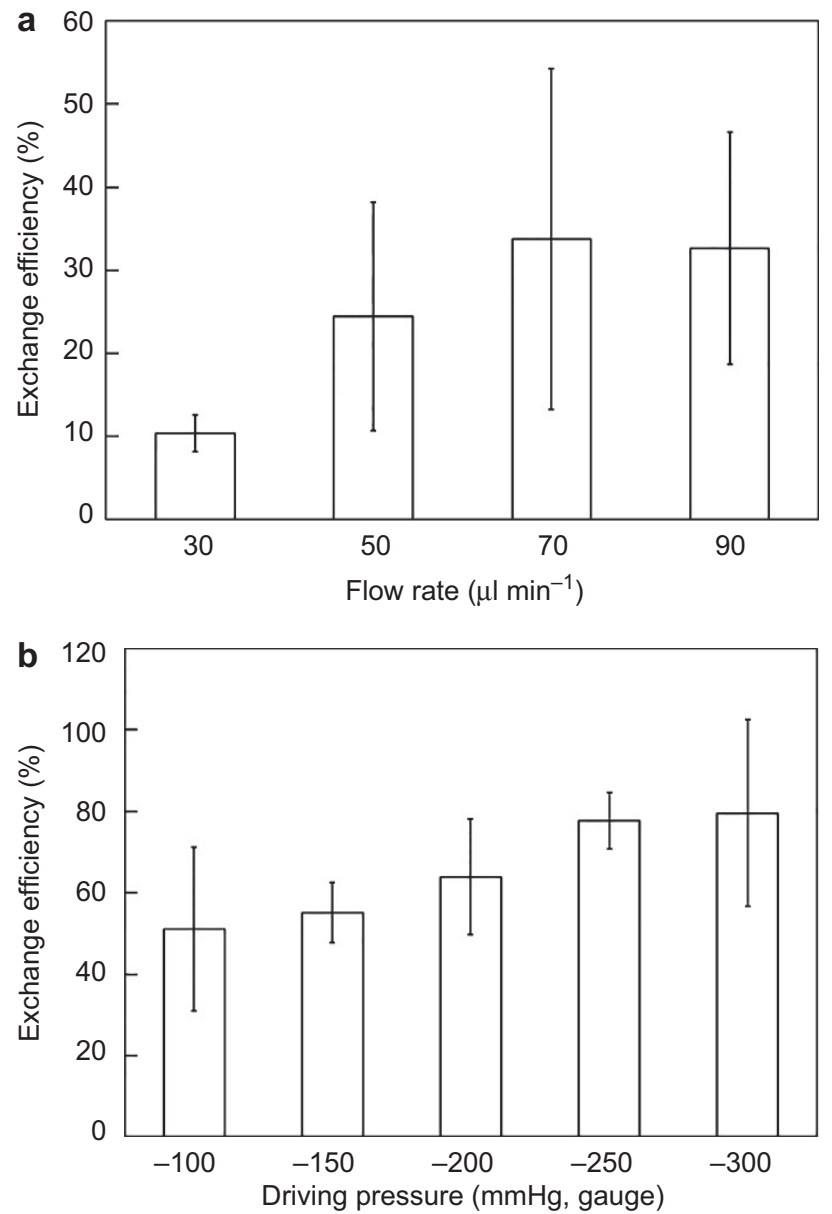

Figure 4 Exchange efficiency of HEK 293T cells processed by the (a) DLD microstructure and the (b) $\mu P A R$ microstructure. (a) The data shows that the exchange efficiency is determined by different input flow rates of the syringe pump. This data suggests that the volume flow rate should be set higher than $90 \mu \mathrm{min}^{-1}$ to achieve the optimal exchange efficiency. (b) The exchange efficiency of the MPAR structure was measured with respect to the negative gauge pressure at the outlet. The device successfully exchanged $79.5 \%$ of the input cells into the electroporation buffer at an applied gauge pressure of $-300 \mathrm{mmHg}$. 
observed at higher flow rates (Figure 4b). The maximum value of the exchange efficiency for the $\mu$ PAR medium exchange module was measured to be $79.5 \%$ at a gauge pressure of $-300 \mathrm{mmHg}$ (Figure 4b). Note that three consecutive experiments were performed for each test. Conventionally, $\mu$ PAR and DLD microstructures were only used to separate mixed particles or cells in single buffer, depending on the size of particles ${ }^{1,31}$. However, MPAR and DLD were used to perform the medium exchange in this study, which represents the first time that these similar microstructures were used for medium exchange.

The effect of the size and the photoconductivity of the virtual electrode on the transmembrane potential

The effects of three independent parameters on the cell transmembrane potential, including the spot diameter of the illuminated pattern, the applied AC frequency and the photoconductivity of the a-Si:H film, were considered when inducing electroporation. Commercially available software (COMSOL Multiphysics, USA) was used to numerically simulate the cell transmembrane potential induced by the virtual electrode. To better understand the relationship between these parameters and the maximum transmembrane potential, the following parameters were used: the relative spot diameter, defined as the full width at half maximum divided by the cell diameter $(12 \mu \mathrm{m})$, and the relative photoconductivity, defined as the a-Si:H photoconductivity divided by the conductivity of the medium outside the cell. The results of the numerical simulation showed that the maximum induced transmembrane potential generally increased with the increasing relative spot diameter and the relative photoconductivity. The dependence of the transmembrane potential on the relative spot diameter can be determined from the pattern of the voltage distribution under different spot diameters (Figure $5 \mathrm{a}$ ), where the relative photoconductivity and the applied AC frequency were 0.1 and $1 \mathrm{kHz}$, respectively. For smaller spot diameters, the cell at the centre of the channel was less affected by the gradient of the voltage distribution across the electroporation buffer. As the diameter of the "virtual electrode" increased, the area of the voltage gradient expanded and reached the cell, inducing a larger transmembrane potential across the cell membrane. To determine the maximum transmembrane potential with respect to the driving frequency, the spot size and the photoconductivity, a simultaneous parametric sweep of all three variables was performed. The size of the black spheres represented the value of the maximum transmembrane potential under each condition (Figure 5b). In general, the induced transmembrane potential at a certain applied peak-topeak voltage could be maximised by increasing the relative photoconductivity and the relative spot diameter, and decreasing the applied AC frequency.

\section{Transfection of plasmid DNA using the integrated microfluidic system}

In our previous work ${ }^{33}$, fluorescence dyes (propidium iodide, PI and calcein acetoxymethyl ester, (aAM) were used to measure the electroporation efficiency when $\mathrm{PPAR}$ microstructures were used to perform the medium exchange. However, using fluorescent dyes to determine the electroporation efficiency is considered to be indirect evidence. In conventional electroporation or transfection experiments, plasmid DNA is commonly used to demonstrate the extent of gene transfection while developing novel methods or new systems ${ }^{34}$. Therefore, the fluorescencecarrying plasmid DNA was used to determine the efficiency of electroporation in this study.

Transfection of a pEGFP-N1 plasmid DNA was conducted to demonstrate the successful integration of the two modules. The use of HEK 293T cells, human embryonic kidney cells, has been a

Relative

spot diameter

Voltege (V)
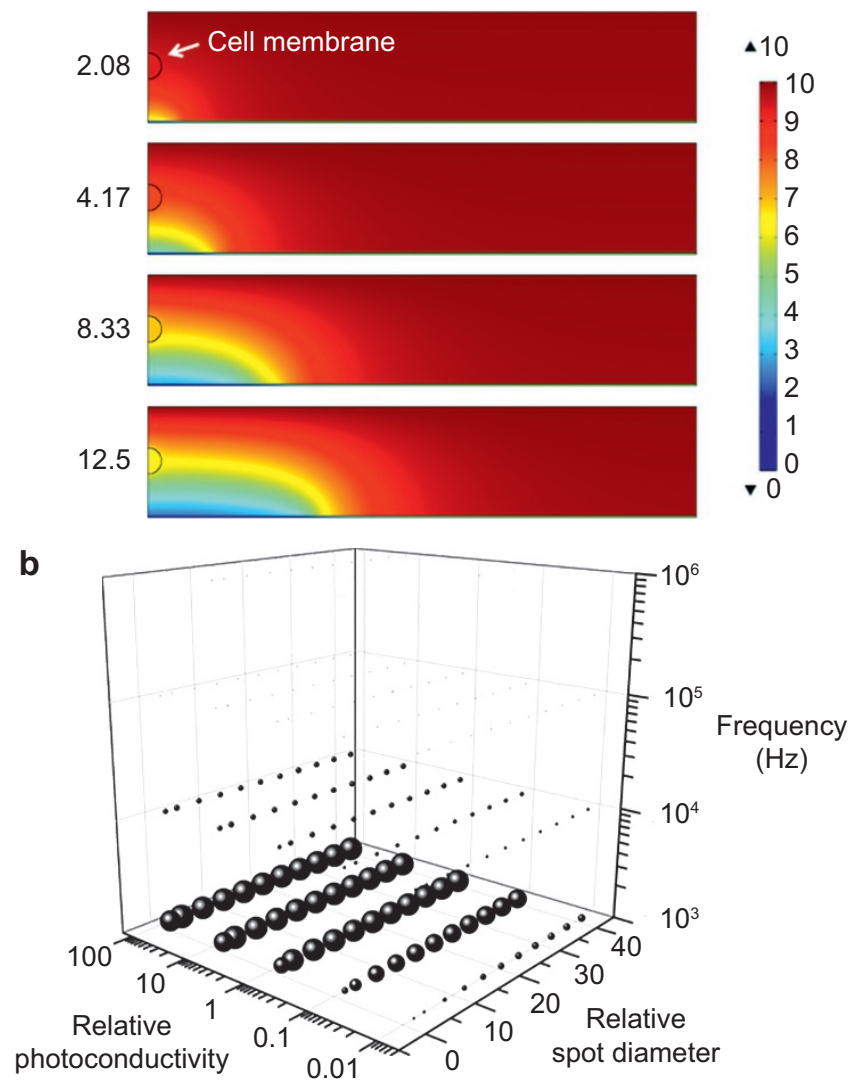

Figure 5 (a) The voltage distribution for different relative spot diameters under a relative photoconductivity of 0.1 and an applied AC frequency of $1 \mathrm{kHz}$. The results indicate that the cell is less affected under smaller relative spot diameters. (b) The OIE simulation results indicate that the transmembrane potential of the cell in the OIE device increased with increasing photoconductivity, larger electrode sizes, and decreasing frequency. The size of the black ball is proportional to the magnitude of the maximum transmembrane potential induced across the cell.

commonly reported for gene transfection and was used as a guideline template for many transfection kits (e.g. lipofectamine, Thermo Fisher Scientific Inc.) $)^{32}$. HEK 293T cells were re-suspended in a culture medium and the electroporation buffer $(0.2 \mathrm{M}$ sucrose) containing the plasmid DNA was injected into the complete integrated system. The 293T cells in the culture medium were transferred into the central stream of the electroporation buffer by the DLD medium exchange module and then were transported to the OIE module for electroporation. The cells then flowed into the OIE zone, where the applied electric field induces a sufficient transmembrane potential across the cell for electroporation. The processing of $250 \mu \mathrm{l}$ of culture medium with suspended cells at a flow rate of $25 \mu \mathrm{l} \mathrm{min}{ }^{-1}$ took approximately $10 \mathrm{~min}$. The fluorescence intensity of the expressed EGFP plasmid was measured using a flow cytometer (BD ACCURI C6, USA). The expressed percentage of green fluorescence signal increased with the increasing applied peak-to-peak voltage in the OIE module. The highest efficiency of $8.3 \%$ occurred at $40 \mathrm{Vpp}$. This efficiency is the highest transfection efficiency ever demonstrated among existing OIE methods ${ }^{19}$. Note that the light irradiance on the OIE zone was $1.5 \mathrm{~W} \mathrm{~cm}^{-2}$ and the volumetric flow rates of

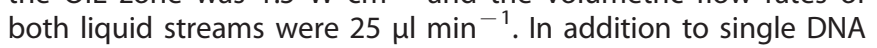



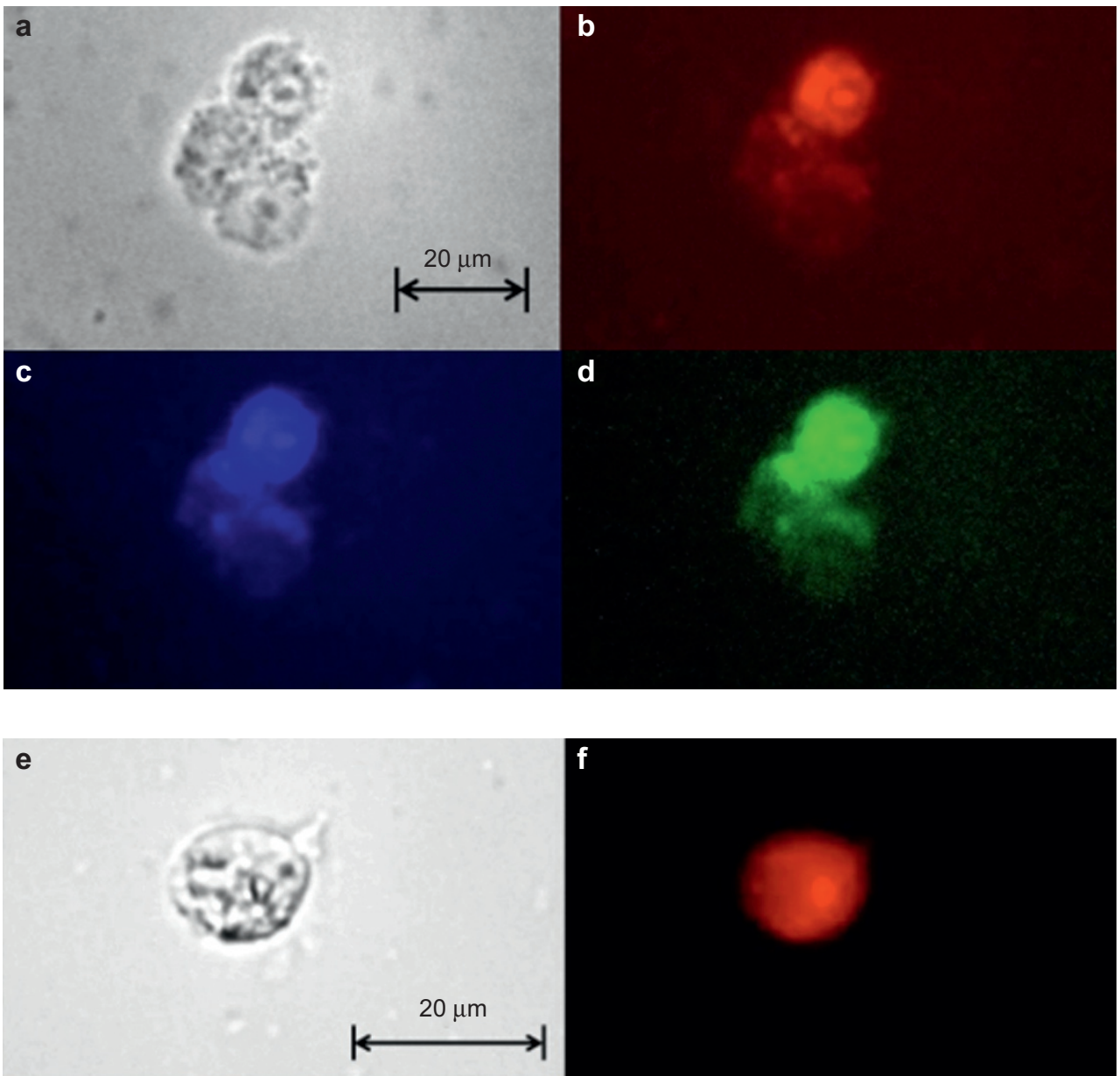

g

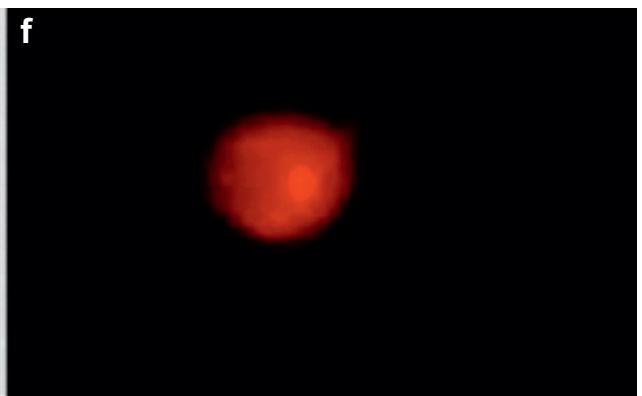

h

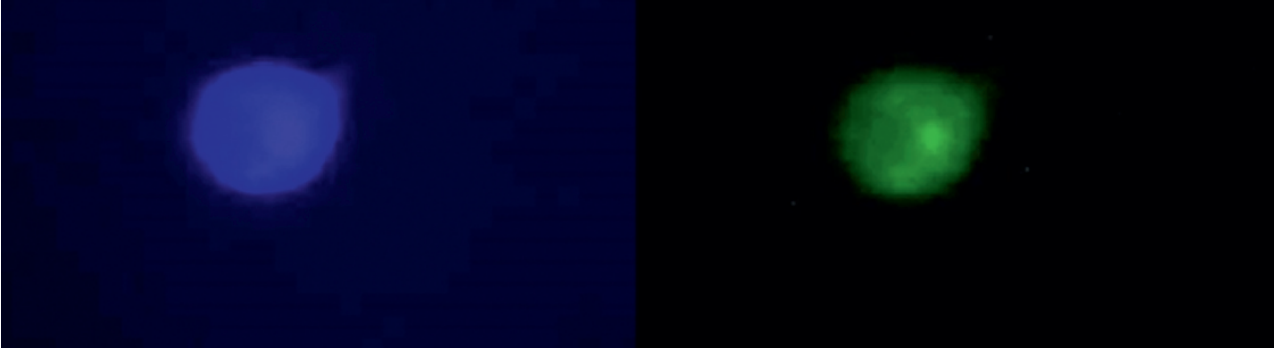

Figure 6 In the OIE module, HEK 293T cells, immersed in the electroporation buffer with plasmid DNA, flowed through the OIE zone (highlighted by the dashed line) and underwent electroporation. The inlet volumetric flow rates of the liquid streams were both $25 \mu$ min ${ }^{-1}$, and the electroporation duration for each cell was approximately $40 \mathrm{~ms}$. The optical pattern was several times larger than the diameter of the cells to facilitate the electroporation of the cells to ensure full coverage of the OIE zone across the channel and to extend the duration for electroporation. A bright field image (a) and fluorescent images (b-d) of an HEK 293T cell 24 hours after the DLD-OIE system treatment. The fluorescent images (f-h) show that three distinct fluorescently labelled plasmids (DsRed, ECFP and EGFP) were successfully cotransfected into a single cell by using the OIE module integrated with the $\mu$ PAR medium exchange module. The volumetric flow rates of the

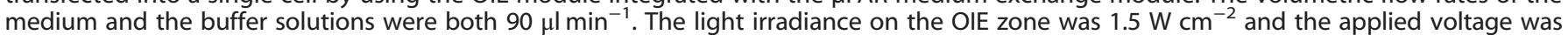
$40 \mathrm{Vpp}$ at a frequency of $20 \mathrm{kHz}$.

transfection, co-transfection of three plasmid DNAs into a single cell was also determined by the developed system, as shown in Figure $6 \mathrm{a}$ and $6 \mathrm{~d}$. The co-transfection into HEK 293T cells of pDsRED, pECFP and pEGFP-N1, which were red, blue and green fluorescently labelled plasmids, respectively, were successfully electroporated by using the DLD-OIE system (Figure $6 \mathrm{~b}-\mathrm{d}$ ) and the $\mu$ PAR-OIE system (Figure 6e-h), as verified by using an upright fluorescence microscope (BX43, Olympus Co., Japan) after
24 hours in culture. Note that the light irradiance on the OIE zone was $1.5 \mathrm{~W} \mathrm{~cm}^{-2}$. The applied voltage was $40 \mathrm{Vpp}$ at a frequency of $20 \mathrm{kHz}$. These images demonstrated that the novel microfluidic system successfully combined continuous medium exchange and optically electroporation modules to automatically perform the entire protocol for the transfection of exogenous molecules from re-suspended cells to electroporation. Note that all cells were viable after the transfection process was completed. 


\section{DISCUSSION}

Cell debris was observed in the channel and between the microposts of the $\mu$ PAR medium exchange module, as well as in the DLD medium exchange module. This debris can reduce the number of cells collected at the central outlet of the medium exchange module and compromise the medium exchange efficiency. The formation of the debris from the disruption of these cells could result from the high fluidic shear stresses experienced during the medium exchange process at high flow rates, where the cells are forced to change their direction in the fluid flow after sliding through the microposts of the $\mu$ PAR structures. The average fluid velocity in this study (approximately $6 \mathrm{~mm} \mathrm{~s}^{-1}$ at a volume flow

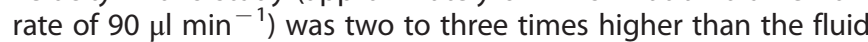
velocity used in the previous study with biological cells $(2.3 \mathrm{~mm}$ $\mathrm{s}^{-1}$ ) where the observed railing failure rates (RFRs) were equivalent to $0 \%$, i.e., no clogging of cells was observed ${ }^{31}$. However, reducing the fluid velocity would compromise the throughput of the medium exchange system and the overall transfection efficiency of the integrated microfluidic system. Therefore, a more thorough study of the relationship between shear-induced cell lysis and the fluid velocity in the medium exchange modules is required in the future. Another factor reducing the cell recovery rate was the cell clogging observed in the $\mu$ PAR or DLD structures during experiments. In the $\mu$ PAR medium exchange module, although the $10 \mu \mathrm{m}$ gaps between microposts were smaller than the cell diameter (13 $\mu \mathrm{m}$ on average for HEK 293T cells), a certain percentage of the cells were still able to squeeze through the gaps or were trapped between posts due to their deformability, hence affecting the module performance. It is believed that the percentage of micropost gaps clogged by cells was inversely related to the volume flow rate. As the cells gained more momentum in the direction of the fluid along the channel, they were less affected by the fluid flowing through the gaps between the microposts, thereby reducing the possibility of being squeezed through or trapped in the gaps. The DLD structure, when compared with the $\mu$ PAR structure, is ideally a clog-free cell separation method because the gaps are larger than the diameter of the cell of interest. However, factors such as variations in cell sizes, clumps of cells adhering together due to poor cell handling before the experiment, and insufficient surface pretreatment and modification to reduce cell adhesion could still result in the clogging of cells, as observed in the medium exchange experiments. Importantly, the cells were always filtered past a cell mesh filter before being injected into the device to ensure that only single cells were allowed into the module. Otherwise, lumps of multiple cells would be trapped in the gaps, thus defeating the purpose of the DLD structure. In addition, a protein-blocking surface treatment was required to prevent the cells from adhering to the PDMS surface because any attached cells would alter the hydrodynamic paths, and therefore adversely affect the cell recovery rate of the medium exchange module.

In this study, we demonstrated a new microfluidic OIE system integrated with the continuous medium exchange module to address the sample pre-treatment problem. The integrated microfluidic system was composed of two separated modules: the continuous medium exchange module (to automatically transfer the re-suspended cells into the specific electroporation buffer) and the OIE module (for cell electroporation). Two microfluidic cell separation methods, DLD and $\mu$ PAR, were used in the design of two continuous medium exchange modules. Experimental results suggest that the $\mu$ PAR structure outperformed the DLD structure in terms of exchange efficiency. This developed microfluidic system is the first integrated microfluidic optical electroporation system capable of performing continuous medium exchange on one device. Compared with previous work using micro-filtering devices for medium exchange, the current work allows for continuous separation of cells and medium exchange, thus increasing the throughput of the developed device ${ }^{20}$. DNA transfection can be performed using microelectrodes. However, microelectrodes require tedious fabrication processes. Both microelectrodes and optically assisted dielectrophoresis on a microsystem could be used for DNA transfection ${ }^{19}$. These approaches require the tested cells to be transfected with extracellular nucleic acids in a "static" situation. Our results represent the first time that the tested cells could be automatically transferred to a suitable transfection buffer and that DNA transfection could be performed in a continuous "flowthrough" format. The throughput of the developed system depended on the volumetric flow rate of the culture medium and the concentration of the cells. For example, the throughput of the integrated microfluidic system was $2.5 \times 10^{4}$ cells per minute for the medium with $10^{6}$ cells ml $^{-1}$ at a volume flow rate of $25 \mu \mathrm{l} \mathrm{min}^{-1}$, assuming no cell loss in the system. The throughput of the system could be increased by adjusting the flow rate and the cell concentration. However, the higher volumetric flow rate would reduce the electroporation duration in the OIE module and may affect the overall electroporation efficiency. Furthermore, the developed device achieved the highest gene transfection efficiency of $8.3 \%$ for EGFP, among all current OIE methods ${ }^{14}$. Moreover, co-transfection of multiple fluorescently labelled plasmids (EGFP, DsRed and ECFP) was confirmed. These results indicate that the developed device has great potential for use in various electroporation-related biological applications, such as creating induced pluripotent stem cells (iPSCs) and for gene therapy.

\section{ACKNOWLEDGEMENTS}

The authors gratefully acknowledge the financial support provided to this study by "the National Science Council in Taiwan (NSC102-2218-E-007-001)". Partial financial support from the "Towards a World-Class University" Project is also greatly appreciated. The authors also thank Dr. Ming-Shiou Jan (Institute of Microbiology and Immunology, College of Medicine, Chung Shan Medical University, Taichung) and Dr. Kuang-Hung Cheng (The Institute of Bio-medical Sciences, National Sun Yat-Sen University, Kaohsiung) for providing the cell line (HEK 293T) and the fluorescently labelled plasmids (pEGFP-N1, pDsRED and pE(FP), respectively.

\section{COMPETING INTERESTS}

The authors declare no conflict of interest.

\section{REFERENCES}

1 Hou HW, Bhagat AAS, Lee WC et al. Microfluidic devices for blood fractionation. Micromachines $2011 ; 2$ : 319-343.

2 Lenshof A, Laurell T. Continuous separation of cells and particles in microfluidic system. Chemical Society Reviews 2010; 39: 1203-1217.

3 Bhagat AAS, Bow $\mathrm{H}$, Hou HW et al. Microfluidics for cell separation. Medical \& Biological Engineering \& Computing 2010; 48: 999-1014.

4 Pamme N. Continuous flow separations in microfluidic devices. Lab on a Chip 2007; 7: 1644-1659.

5 Xuan X, Zhu J, Church C. Particle focusing in microfluidic devices. Microfluid and nanofluid 2010; 9: 1-16.

6 Movahed S, Li D. Microfluidics cell electroporation. Microfluid and nanofluid 2011; 10: 703-734.

7 Kim J, Hwang I, Britan D et al. Microfluidic approaches for gene delivery and gene therapy. Lab on a Chip 2011; 11: 3941-3948.

8 Wang M, Orwar O, Olofsson J et al. Single-cell electroporation. Analytical and Bioanalytical Chemistry 2010; 397: 3235-3248.

9 Potter H, Heller R. Transfection by electroporation. Current Protocols in Neuroscience 2011; Appendix 1: $1 \mathrm{E}$.

10 Jordan ET, Collins M, Terefe J et al. Optimizing electroporation conditions in primary and other difficult-to-transfect cells. Journal of Biomolecular Techniques 2008; 19: 328-334.

11 Pucihar G, Kotnik T, Kandušer $M$ et al. The influence of medium conductivity on electropermeabilization and survival of cells in vitro. Bioelectrochemistry 2001; 54: 107-115.

12 Lee WG, Demirci U, Khademhosseini A. Microscale electroporation: Challenges and perspectives for clinical applications. Integrative Biology 2009; 1: 242-251. 
13 Fei $Z$, Wang $S$, Xie $Y$ et al. Gene transfection of mammalian cells using membrane sandwich electroporation. Analytical Chemistry 2007; 79: 5719-5722.

14 Valero A, Post JN, van Nieuwkasteele JW et al. Gene transfer and protein dynamics in stem cells using single cell electroporation in a microfluidic device. Lab on a Chip 2008; 8: 62-67.

15 Valley JK, Neale S, Hsu HY et al. Parallel single-cell light-induced electroporation and dielectrophoretic manipulation. Lab on a Chip 2009; 9: 1714-1720.

16 Wang HY, Lu C. Electroporation of mammalian cells in a microfluidic channel with geometric variation. Analytical Chemistry 2006; 78: 5158-5164.

17 Zhan Y, Wang J, Bao N et al. Electroporation of cells in microfluidic droplets. Analytical Chemistry 2009; 81: 2027-2031.

18 Lin YH, Lee GB. An optically induced cell lysis device using dielectrophoresis. Applied Physics Letters 2009; 94: 033901.

19 Wang $\mathrm{CH}$, Lee $\mathrm{YH}$, Kuo HT et al. Dielectrophoretically-assisted electroporation using light-activated virtual microelectrodes for multiple DNA transfection. Lab on a Chip 2014; 14: 592-601.

20 Lee GB, Wu HC, Yang PF et al. Optically induced dielectropheresis sorting with automated medium exchange in an integrated optofluidic device resulting in higher cell viability. Lab on a Chip 2014; 14: 2837-2843.

21 Beech JP, Holm SH, Adolfsson $\mathrm{K}$ et al. Sorting cells by size, shape and deformability. Lab on a Chip 2012; 12: 1048-1051.

22 Huang LR, Cox EC, Austin RH et al. Continuous particle separation through deterministic lateral displacement. Science 2004; 304: 987-990.

23 Holm SH, Beech JP, Barrett MP et al. Separation of parasites from human blood using deterministic lateral displacement. Lab on a Chip 2011; 11: 1326-1332.

24 Inglis DW, Davis JA, Austin RH et al. Critical particle size for fractionation by deterministic lateral displacement. Lab on a Chip 2006; 6: 655-658.

25 Davis JA, Inglis DW, Morton KJ et al. Deterministic hydrodynamics: Taking blood apart. Proceedings of the National Academy of Sciences of the United States of America PNAS, Proceedings of the National Academy of Sciences 2006; 103: 1477914784.
26 Morton $\mathrm{KJ}$, Loutherback $\mathrm{K}$, Inglis DW et al. Hydrodynamic metamaterials: Microfabricated arrays to steer, refract, and focus streams of biomaterials. Proceedings of the National Academy of Sciences of the United States of America PNAS, Proceedings of the National Academy of Sciences 2008; 105: 7434-7438.

27 Green JV, Radisic M, Murthy SK. Deterministic lateral displacement as a means to enrich large cells for tissue engineering. Analytical Chemistry 2009; 81: 9178-9182.

28 David WI, Megan L, Robert EN. Scaling deterministic lateral displacement arrays for high throughput and dilution-free enrichment of leukocytes. Journal of Micromechanics and Microengineering 2011; 21: 054024.

29 Liu Z, Huang F, Du J et al. Rapid isolation of cancer cells using microfluidic deterministic lateral displacement structure. Biomicrofluidics 2013; 7: 011801.

30 Karabacak NM, Spuhler PS, Fachin F et al. Microfluidic, marker-free isolation of circulating tumor cells from blood samples. Nature Protocols 2014; 9: 694-710.

31 Sochol RD, Li S, Lee LP et al. Continuous flow multi-stage microfluidic reactors via hydrodynamic microparticle railing. Lab on a Chip 2012; 12: 4168-4177.

32 Sochol RD, Corbett $\mathrm{D}$, Hesse $\mathrm{S}$ et al. Dual-mode hydrodynamic railing and arraying of microparticles for multi-stage signal detection in continuous flow biochemical microprocessors. Lab on a Chip 2014; 14: 1405-1409.

33 Chang CJ, Lu MY, Lee GB. A continuous optically-induced cell electroporation device with on-chip medium exchange mechanisms. IEEE 27th International Conference on Micro Electro Mechanical Systems (IEEE MEMS); 26-30 Jan 2014; San Francisco, CA, USA; 2014: 234-237.

34 Le Gac S, van den Berg A. Single cell electroporation using microfluidic devices. Methods in Molecular Biology 2012; 853: 65-82.

(c) (1) $(-)$ This license allows readers to copy, distribute and transmit the Contribution as long as it attributed back to the author. Readers may not alter, transform or build upon the Contribution, or use the article for commercial purposes. Please read the full license for further details at - http://creativecommons. org/licenses/by-nc-nd/4.0/ 